\title{
Troubleshooting in Upgrading Procedure: Intraprocedural Percutaneous Transluminal Angioplasty of Great Vessels
}

\author{
Morani G*, Bolzan B, Zimelli E, Tomasi L and Ribichini FL \\ Department of Medicine, University of Verona, Italy \\ *Corresponding author: Giovanni Morani, Division of Cardiology, University of Verona, Italy
}

\begin{abstract}
Upgrading procedure is relatively common since indications to cardiac resynchronization therapy (CRT) and implantable defibrillator are increasing. A relatively frequent phenomenon that the operator may encounter is some degree of venous obstruction around the previously implanted leads, which may become completely occlusive. In these situations, upgrading procedure may be challenging and various manoeuvers have been described. We describe a case of intraprocedural percutaneous transluminal angioplasty (PTA) of occlusive stenosis of the innominate vein, performed during an upgrading procedure from bicameral cardiac implantable defibrillator (ICD) to CRT-D.
\end{abstract}

Keywords: Upgrading; Cardiac resynchronization therapy; Percutaneous transluminal angioplasty; Venous occlusion

\section{Introduction}

Cardiac resynchronization therapy is a well-established therapy for patients with severe systolic dysfunction, wide QRS and heart failure despite optimal medical therapy [1]. Moreover, since indications for cardiac resynchronization devices continue to expand, the number of upgrading procedures from previously implanted single- or dual-chamber systems is increasing. In these cases, some degree of venous obstruction is a relatively frequent phenomenon (up to 50\%), which may become completely occlusive in $12 \%$ of cases [2]. Chronic venous occlusion, mostly asymptomatic, can involve the coronary sinus and its branches as well as central veins, such as the axillary-subclavian or innominate veins. Angioplasty of the coronary sinus is well described in the literature to allow the placement of a left ventricular lead (LV) $[3,4]$. By contrast, not many data are available on the intra-procedural safety and efficacy of central vessel angioplasty [5,6]. We describe a successful case of angioplasty of a total central venous occlusion to allow LV lead placement during an upgrading procedure to a CRT system.

\section{Case Report}

A 69-year-old patient with a clinical history of hypertension, diabetes, prior myocardial infarction, poor EF (30\%), and previous percutaneous transluminal coronary angioplasty (PTCA) on the circumflex coronary artery, was referred to our institution for elective replacement of a dual-chamber ICD implanted in July 2008 (ICD Lumos DRT, atrial lead Selox JT 53 and ventricular lead Linox SD 65/16, Biotronik SE. Berlin Germany). On admission, the patient was symptomatic for dyspnea, with a poor functional class (NYHA III), the echocardiogram showed a severely depressed ejection fraction (20\%) and the electrocardiogram confirmed a wide QRS duration of $130 \mathrm{msec}$ with left bundle branch block. A coronary angiography confirmed the indication to medical therapy in the absence of any target vessel for revascularization. We consequently decided to perform an upgrading procedure to a CRT system. In accordance with our clinical practice, angiography of the axillary and subclavian veins through the ante cubital venous access was performed before the procedure to assess patency of the central 
veins. The angiogram documented severe stenosis of the axillary vein at the insertion point of the right atrial and ventricular leads into the vessel. The subclavian vein was then directly punctured distally to the sub-occlusive stenosis (behind first rib), to obtain a venous access for the LV lead. Unfortunately, subsequent advancing on the 0.035 " guidewire through a sealed $9 \mathrm{Fr}$ introduction sheath (Safe Sheath HLS 1009, Pressure Product, San Pedro, USA) proved unsuccessful owing to another occlusion at the junction between the superior vena cava (SVC) and the innominate vein, where the second shocking coil lead was located. Selective injection of contrast media into the subclavian vein through the introducer confirmed total occlusion of the main vessel and a wide collateral vicarious circulation draining blood into the SVC (Figure 1).

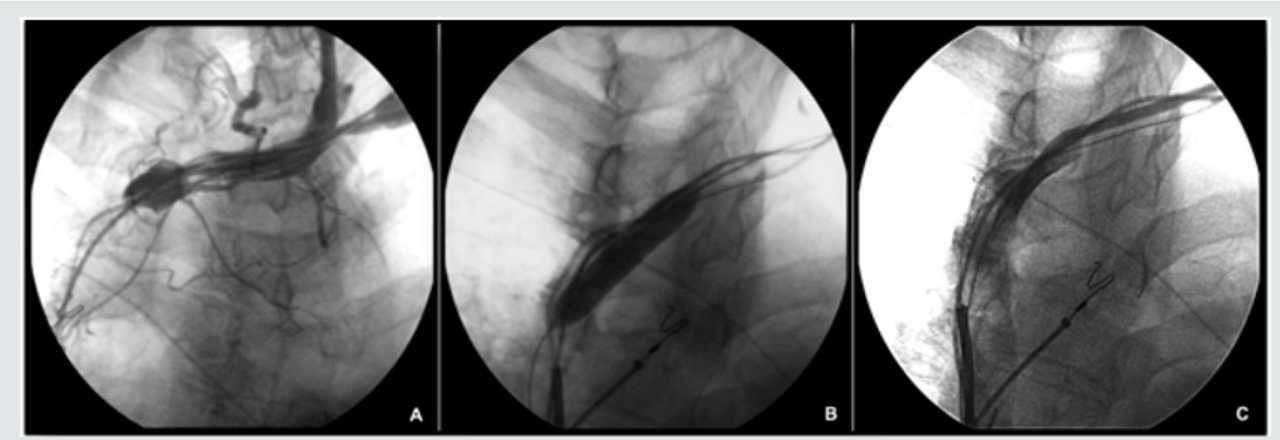

Figure 1: Vein angioplasty procedure. A. Angiography from the subclavian venous access, revealing occlusive venous stenosis of the anonimous trunk. B. Balloon inflated up to 8-10 atm. across the stenosis. C. Restoration of venous patency after angioplasty.

A 0.014" angioplasty guidewire (TERUMO Corporation, Tokyo, Japan) was threaded through the occlusion and then left in the right atrium. An angioplasty balloon (Sterling $6 \mathrm{~mm} \times 20 \mathrm{~mm}$, Boston Scientific Inc. Natick, USA) was then advanced over the wire to the occlusion and inflated to $6 \mathrm{~atm}$. In order to progressively open the occlusion, we removed the balloon and advanced over the 0.014 " wire a multipurpose catheter (Cordis Corporation, Miami, USA) which, thanks to the dotter effect, enabled us to pass through the stenotic tract and to replace the 0.014 " with a 0.35 " x $200 \mathrm{~cm}$ guidewire. Once we had retrieved the MP catheter, a larger angioplasty balloon (Sterling $8 \mathrm{~mm}$ x $40 \mathrm{~mm}$, Boston scientific Inc. Natick, USA) was advanced over the 0.35 " wire and repeatedly inflated to 10 atm to obtain satisfactory dilation of the subclavian and innominate veins (Figure 1B). A 5-10 cc injection of contrast medium confirmed the re-establishment of flow within the subclavian vein and SVC, with a residual stenosis of 30-40\% (Figure 1C). Implantation of the CRT device was then successfully carried out, without complications, by cannulating the coronary sinus through a dedicated delivery system (Attain Command Straight, Medtronic Inc. Minneapolis, USA), placing a 4 Fr dual-unipolar LV lead (Attain Ability 4196$88 \mathrm{~cm}$, Medtronic Inc. Minneapolis, USA) into a lateral coronary vein and, finally, by connecting the three leads to a CRT-D device (Consulta CRT-D, Medtronic Inc. Minneapolis, USA). The acute $\mathrm{LV}$ lead threshold was $1.1 \mathrm{~V}$ at $0.5 \mathrm{msec}$, with no diaphragmatic capture at $10 \mathrm{~V}$. The electrical parameters of the right atrial and right ventricular leads were also stable. The additional time required to perform PTA was 15 minutes, plus 3 minutes of fluoroscopy time with an additional $30 \mathrm{cc}$ injection of contrast medium injection. At the 3-month follow-up examination, electrical parameters of atrial, right ventricle and left ventricle leads were stable, and no adverse event was observed.

\section{Discussion}

Chronic venous occlusion is not an infrequent finding in patients undergoing implantation procedures. This issue may involve both the coronary sinus and the central venous system. Recognized risk factors for venous obstruction include blood stasis due to venous angulation or kinking, the presence of a central venous line for long-term infusion therapy, hemodialysis, trauma, infection and the presence of pacemaker leads. Heart failure and poor systolic function may facilitate the process $[7,8]$. The most likely pathogenesis seems to be due to thrombosis, beginning with fibrin deposition over the lead surface and progressive fibrous reaction, with a ring-like fibrosis development around the lead, causing severe stenosis or even total occlusion of the vein [5]. The presence of a second ICD shocking coil, usually located in the superior vena cava, has been associated with increased incidence of venous obstruction [9]. In our case, the occlusion was localized at the junction of the innominate vein with the superior vena cava, where the second shocking coil lead lays for anatomical reasons. Thrombosis and fibrotic reaction were probably favored by the close contact of the heavy coil with the vessel wall at a point where, for hemodynamic reasons, there may be turbulence, thereby predisposing the vessel to occlusive stenosis.

As the stenotic process is usually gradual, a collateral venous network can develop to compensate for the occlusion. Consequently, symptoms or physical signs are rare, and partial or total venous occlusion may be totally asymptomatically, becoming an unexpected finding at the time of implantation. If a new lead needs to be added to the implanted system, the operative strategy may differ considerably according to the physician's familiarity with the available technical options. The physician can choose 
among various approaches: an opposite-side approach with subsequent lead tunneling across the thorax [10], an ipsilateral approach performed by means of jugular system cannulation [11], and epicardial placement of the leads through thoracotomy or vein angioplasty [5]. The first option carries several risks, including the development of complete occlusion of both subclavian veins over time, damage to the leads - particularly deleterious in the case of ICD leads - or higher defibrillation threshold in the case of rightside placement of the active can. The jugular system approach may expose the patient to the risk of worsened blood drainage or, in the case of the internal jugular cannulation, even acute serious damage to this important vessel of the neck [12]. Epicardial placement of the leads through thoracotomy has the disadvantage of requiring surgery and general anesthesia.

Our experience showed that angioplasty of the central venous system can be performed in the same session of the upgrading procedure without excessively prolonging the procedural time, thereby avoiding postponing the procedure. We observed no complications, damage or dislocations of the existing leads. Moreover, this approach is less invasive than other alternatives and allows the patency of the contralateral venous system to be preserved. This case also shows that in certain clinical conditions at higher risk of venous occlusion, it is advisable to perform an angiography from ante cubital vein before the procedure, in order to assess the patency and exact location of the great veins. We can hypothesize that incomplete location of the second shocking coil inside the superior vena cava and the lying of the coil across the junction between the innominate vein and superior vena cava may contribute, for hemodynamic reasons, to the development of a marked fibrotic reaction. Eventually, in case of occlusive stenosis of central veins, intraprocedural venoplasty of great vessel seem to be feasible, without postponing the upgrading procedure.

\section{References}

1. Piotr Ponikowski, Adriaan A Voors, Stefan D Anker, Héctor Bueno, John G F Cleland, et al. (2016) ESC Guidelines for the diagnosis and treatment of acute and chronic heart failure: The Task Force for the diagnosis and treatment of acute and chronic heart failure of the European Society of Cardiology (ESC) Developed with the special contribution of the Heart Failure Association (HFA) of the ESC. European Heart Journal 37(27): 2129-2200.

2. Sticherling C, Cough SP, Baker RL, Wasmer K, Orai FI, et al. (2001) Prevalence of centrai venous occlusion in patients with chronic defibrillator leads. Am Heart J 141(5): 813-816.

3. Flansky B, Lamp B, Minami K, Fleintze J, Krater L, et al. (2002) Coronary vein balloon angioplasty for left ventricular pacemaker lead implantation. J Am Coll Cardiol 40(12): 2144-2149.

4. Kowalski O, Lenarczyk R, Procopczuk J, Pruszkowska Skrzep P, et al. (2006) Effect of percutaneous interventions within the coronary sinus on the success rate of the implantation of resynchronization pacemakers. Pace 29(10): 1075-1080.

5. McCotter CJ, Angle JF, Prudente LA, Mounsey JP, Ferguson JD, et al. (2005) Placement of transvenous pacemakers and ICD leads across total chronic occlusions. Pace 28(9): 921-925.

6. Luerdorff G, Grove R Kranig W, Thale I (2009) Different venous angioplasty manoeuvres for successful implantation of CRT devices. Clin Res Cardiol 98(3): 159-164.

7. Da Costa SS, Scalabrini Neto A, Costa R, Caldas JG, Martinelli Filho M (2002) Incidence and Risk Factors of Upper Extremity Deep Vein Lesions After Permanent Transvenous Pacemaker Implant: A 6-Month Follow-Up Prospective Study. Pace 25(9): 1301-1306.

8. Oginosawa Y, Abe H, Nakashima Y (2005) Prevalence of Venous Anatomic Variants and Occlusion Among Patients Undergoing Implantation of Transvenous Leads. Pace 28(5): 425-428.

9. Lickfett L Bitzen A, Arepally A, Nasir K, Wolpert C, et al. (2004) Incidence of venous obstruction following insertion of an impiantale cardioverter defibrillator. A study of systematic contrast venography on patients presenting for their first elective ICD generator replacement. Europace 6(1): $25-31$

10. Fox DJ, Petkar S, Davidson NC, Fitzpatrick AP (2006) Upgrading patients with chronic defibrillator leads to a biventricular System and reducing patient Risk: contralateral LV lead placement. Pace 29(9): 1025-1027.

11. Bosa Ojeda F, Bethencourt Munoz M, Vargas Torres M, Lara Paoron A, Rodriguez Gonzalez A, et al. (2007) Upgrade of a pacemaker defibrillator to a biventricular device: The internal jugular vein approach in a case of bilateral subclavian vein occlusion. J Interv Card Electrophysiol 19(3): 209-211.

12. Antonelli D, Freedberg NA, Rosenfeld T (2001) Lead insertion by supraclavicular approach of the subclavian vein puncture. Pace 24(3): 379-380.

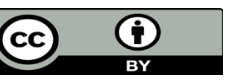

This work is licensed under Creative Commons Attribution 4.0 License

To Submit Your Article Click Here: Submit Article

DOI: 10.32474/ACR.2019.02.000128

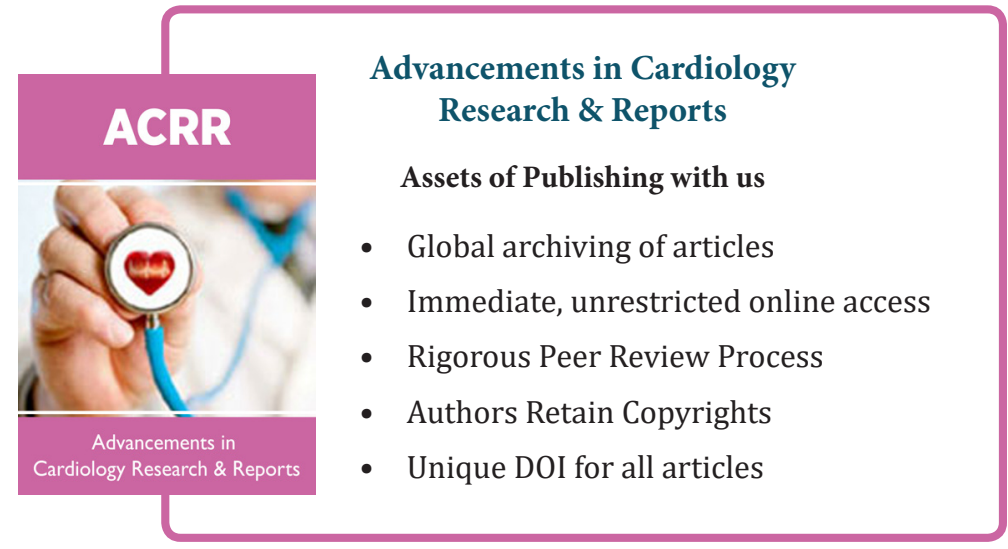

